\title{
Renal Cell Carcinoma with Inferior
}

\section{Vena Cava Extension: Can Classification Be Optimized to Predict Perioperative Outcomes?}

\author{
Bradley C. Leibovich ${ }^{\mathrm{a}}$, Christine M. Lohse ${ }^{\mathrm{b}}$, John C. Cheville ${ }^{\mathrm{c}}$, Theodora A. Potretzke ${ }^{\mathrm{d}}$, \\ Matvey Tsivian ${ }^{\mathrm{a}}$, Paras H. Shah ${ }^{\mathrm{e}}$, Stephen A. Boorjian ${ }^{\mathrm{a}}$, R. Houston Thompson ${ }^{\mathrm{a}}$ \\ and Timothy D. Lyon ${ }^{\mathrm{f}, *}$ \\ ${ }^{a}$ Department of Urology, Mayo Clinic, Rochester, MN, USA \\ ${ }^{\mathrm{b}}$ Department of Health Sciences Research, Mayo Clinic, Rochester, MN, USA \\ ${ }^{\mathrm{c}}$ Department of Laboratory Medicine and Pathology, Mayo Clinic, Rochester, MN, USA \\ ${ }^{\mathrm{d}}$ Department of Radiology, Mayo Clinic, Rochester, MN, USA \\ e Division of Urology, Albany Medical College, Albany, NY, USA \\ ${ }^{\mathrm{f}}$ Department of Urology, Mayo Clinic, Jacksonville, FL, USA
}

Received 17 August 2019

Accepted 9 March 2020

Pre-press 14 April 2020

Published 2 July 2020

\begin{abstract}
Ambiguity exists regarding the definition of a level III inferior vena cava tumor thrombus (IVC-TT), limiting comparisons between open and minimally-invasive series. We assessed 253 patients who underwent radical nephrectomy with IVC-TT from 2000-2015 and proposed a modified classification based on associations between intraoperative IVC clamp position and need for cardiopulmonary bypass with complications, length of stay, and blood transfusions. Predictive ability of the modified system was not meaningfully improved (AUCs $0.59-0.58 ; 0.61-0.61 ; 0.72-0.72$ ). Nevertheless, we advocate for standardization of the border of a level III thrombus at or above the major hepatic veins to facilitate meaningful comparisons between techniques.
\end{abstract}

Keywords: Renal cell carcinoma, venous tumor thrombus, thrombectomy, complications, classification

\section{INTRODUCTION}

The Mayo Clinic thrombus classification is widely used to describe levels of inferior vena cava tumor thrombus (IVC-TT) in patients with locally advanced renal cell carcinoma (RCC) [1]. However, there remains ambiguity in the literature as to the

${ }^{*}$ Correspondence to: Timothy D. Lyon, MD, Mayo Clinic, 4500 San Pablo Road, Jacksonville, FL 32224, USA. Tel.: +1 9049532000; E-mail: lyon.timothy@mayo.edu. precise definition of a level III thrombus, with some authors defining thrombus above the short hepatic venous branches from the caudate lobe as level III $[2,3]$, while others utilize extension to or above the major hepatic veins to define level III [1, 4]. This discrepancy has particular relevance in the context of several recent reports of robotic-assisted radical nephrectomy with IVC thrombectomy [2, $3]$. Given the increased surgical complexity of managing tumors above the major hepatic veins, wherein liver mobilization, hepatic inflow occlusion, 
or supradiaphragmatic IVC control may be required, there is a clear need to standardize definitions of level II and III thrombi to allow for more precise and meaningful comparisons between surgical approaches.

Furthermore, the existing Mayo thrombus classification was developed based on anatomic landmarks as well as an admittedly arbitrary $2 \mathrm{~cm}$ distance from the ostium of the renal vein, and whether successive levels of IVC involvement correlate with perioperative morbidity remains to be characterized. Indeed, maneuvers such as hepatic mobilization or ligation of the short hepatic venous branches might serve as a more relevant metric to inform postoperative complication risk than the length of linear thrombus ascent. Herein, we hypothesized that specific operative maneuvers performed during radical nephrectomy with IVC thrombectomy would be associated with 30-day morbidity, and that these maneuvers could be used to modify the IVC-TT classification to optimize the prediction of perioperative outcomes.

\section{MATERIALS AND METHODS}

After Institutional Review Board approval (IRB\# 13-006722), we identified RCC patients with IVC thrombus treated at a single institution by 11 different surgeons in our Nephrectomy Registry from 2000-2015, and divided into training (2000-2010) and validation (2011-2015) cohorts. Patients with a solitary kidney $(n=6)$, missing imaging $(n=23)$, or direct extramural invasion of the IVC $(n=1)$ were excluded, leaving 166 and 87 patients in the training and validation cohorts, respectively.

Operative reports were reviewed to ascertain the following details: operative time, incision type, sternotomy, IVC clamp position, need for division of short hepatic veins, contralateral renal vein control, hepatic inflow occlusion, hepatic mobilization, cardiopulmonary or venovenous bypass, circulatory arrest, venous reconstruction, and pulmonary embolectomy. One investigator blinded to patient outcome (TDL) reviewed all operative reports, while another (BCL), who was one of the operating surgeons, reviewed a $15 \%$ random sample to assess inter-rater agreement. All specimens were reviewed by a single pathologist (JCC), and are reported according to the 2018 tumor, node, metastasis stage and International Society of Urological Pathology grade classifications. The presence of viable cancer cells within the thrombus is not reported as this information is not captured in our registry.

The perioperative outcomes of interest included any complication within 30 days of surgery, prolonged length of hospital stay ( $\geq 75$ th percentile), and increased volume of blood transfused (units $\geq 75$ th percentile). Univariable associations between operative maneuvers and perioperative outcomes in the training cohort were examined to develop a modified thrombus classification intended to describe the expected IVC clamp position relative to the liver in an effort to account for differences in surgical complexity. Preoperative radiographic images, consisting of magnetic resonance imaging and/or computed tomography scans, were re-reviewed by one radiologist (TAP) to reclassify according to the modified definition. All scans were found to be of adequate quality to permit reclassification. The predictive ability of the current and modified thrombus classifications was summarized with the area under a receiver operating characteristics curve (AUC).

\section{RESULTS}

Clinical features and demographics of patients in the training cohort are summarized in Table 1, and a comparison of features in the training and validation cohorts in Supplementary Table 1. The distribution of current IVC-TT levels in the training cohort included level I in 53 (32\%), II in 62 (37\%), III in $25(15 \%)$, and level IV in $26(16 \%)$. Inter-rater agreement for operative variables was excellent (kappa 0.78-1.0). Complications occurred in $52(31 \%)$ and 29 (33\%) patients in the training and validation cohorts, respectively. Univariable associations between candidate operative maneuvers and perioperative outcomes can be found in Supplementary Table 2. Maneuvers with the highest predictive ability for the three perioperative outcomes of any complication within 30 days, prolonged length of hospital stay, and increased volume of blood transfused included retro- or suprahepatic IVC clamp position (AUCs $0.62,0.63,0.75$ ) and need for cardiopulmonary bypass (AUCs 0.55, $0.61,0.72$ ).

A modified thrombus classification was thereby proposed, with successive levels defined as follows (Fig. 1): level I (into IVC below inferior margin of the caudate lobe, suggesting need for subhepatic IVC clamp), level II (above caudate and below major hepatic veins, suggesting need for retrohepatic IVC clamp), level III (at or above major hepatics, 
Table 1

Clinical and pathologic features of the training cohort, $N=166$

\begin{tabular}{|c|c|}
\hline Feature & Mean (SD) \\
\hline Age, years & $63.6(10.5)$ \\
\hline Body mass index, $\mathrm{kg} / \mathrm{m}^{2}(N=163)$ & $30.0(6.0)$ \\
\hline Tumor size, $\mathrm{cm}$ & $11.0(4.4)$ \\
\hline AP diameter of IVC at $\mathrm{RVo}, \mathrm{mm}(N=163)$ & $26.1(8.8)$ \\
\hline \multirow[t]{2}{*}{ Operative time in hours } & $5.0(2.1)$ \\
\hline & Median (IQR) \\
\hline \multirow[t]{2}{*}{ Charlson Comorbidity Index } & $1(0-6)$ \\
\hline & $\mathrm{N}(\%)$ \\
\hline Female Sex & $48(29)$ \\
\hline \multicolumn{2}{|l|}{ ECOG performance status $(N=165)$} \\
\hline 0 & $113(68)$ \\
\hline 1 & $26(16)$ \\
\hline 2 & $19(12)$ \\
\hline 3 & $6(4)$ \\
\hline 4 & $1(1)$ \\
\hline \multicolumn{2}{|l|}{ Smoking history $(N=164)$} \\
\hline Never & $59(36)$ \\
\hline Current & $38(23)$ \\
\hline Former & $67(41)$ \\
\hline Hemoglobin below LLN & $118(71)$ \\
\hline Calcium above ULN $(N=111)$ & $22(20)$ \\
\hline Platelets above ULN $(N=150)$ & $18(12)$ \\
\hline Preoperative systemic therapy & $5(3)$ \\
\hline Preoperative VTE $(N=163)$ & $18(11)$ \\
\hline \multicolumn{2}{|l|}{ Preoperative symptoms: } \\
\hline Lower extremity edema $(N=160)$ & $41(26)$ \\
\hline Claudication $(N=162)$ & $12(7)$ \\
\hline Varicocele $(N=162)$ & $16(10)$ \\
\hline Scrotal/groin swelling $(N=162)$ & $7(4)$ \\
\hline Right sided tumor & $107(64)$ \\
\hline AP diameter of IVC at $\mathrm{RVo} \geq 24.0 \mathrm{~mm}(N=163)$ & $95(58)$ \\
\hline Complete occlusion of IVC at RVo $(N=164)$ & $37(23)$ \\
\hline $\mathrm{cN} 1$ & $45(27)$ \\
\hline Lymph node dissection & $132(80)$ \\
\hline M1 & $51(31)$ \\
\hline Concurrent metastasectomy & $21(13)$ \\
\hline Additional surgical procedure(s) & $54(33)$ \\
\hline \multicolumn{2}{|l|}{ Histologic subtype } \\
\hline Clear cell RCC & $146(88)$ \\
\hline Papillary RCC & $7(4)$ \\
\hline Clear cell papillary RCC & $1(1)$ \\
\hline Collecting duct RCC & $3(2)$ \\
\hline Unclassified RCC & $9(5)$ \\
\hline \multicolumn{2}{|l|}{ Grade } \\
\hline 1 & 0 \\
\hline 2 & $5(3)$ \\
\hline 3 & $87(52)$ \\
\hline 4 & $74(45)$ \\
\hline Coagulative tumor necrosis & $130(78)$ \\
\hline Sarcomatoid differentiation $(N=165)$ & $26(16)$ \\
\hline \multicolumn{2}{|l|}{ Current tumor thrombus level } \\
\hline I & $53(32)$ \\
\hline II & $62(37)$ \\
\hline III & $25(15)$ \\
\hline IV & $26(16)$ \\
\hline
\end{tabular}

RVo renal vein ostium; ECOG Eastern Cooperative Oncology Group; IVC inferior vena cava; $L L N$ lower limit of normal; $U L N$ upper limit of normal; VTE venous thromboembolism. suggesting need for suprahepatic, infradiaphragmatic IVC clamp and possible hepatic inflow occlusion), and level IV (above diaphragm, requiring supradiaphragmatic IVC clamp or bypass). Patients were then reclassified based upon the modified definition; specifically, 39 of the $62(63 \%)$ patients in the training cohort currently classified as level II were re-classified as level I. Despite this reclassification, predictive ability of the current and modified thrombus classifications with outcomes was similar in both the training and validation cohorts, as shown in Table 2.

\section{DISCUSSION AND CONCLUSIONS}

The purpose of this study was to assess whether intraoperative maneuvers were associated with outcome following radical nephrectomy with IVC-TT, and to propose a modified Mayo classification system based on the findings. The data presented here do not support a change to the classification system, as predictive ability for perioperative morbidity was not meaningfully improved. Nevertheless, there remains a need for standardization of IVC-TT level to facilitate accurate comparisons between surgical techniques. As extension to the level of the major hepatic veins has important implications for operative resource planning - such as need for suprahepatic IVC control or hepatic inflow occlusion- it is our position that a level III thrombus should be definitively classified as one that reaches the major hepatic veins.

The text of the original definition by Neves and Zincke is vague, describing a level III as extension into the intrahepatic vena cava but below the diaphragm [1]. Moreover, Fig. 1 from that manuscript unfortunately does not match the authors' text description of a level III thrombus, and may be misleading. This discrepancy is overlooked unless the captions for subsequent figures are scrutinized. Specifically, Fig. 1 depicts an intrahepatic thrombus (level III) as extending above the caudate lobe but below the major hepatic veins; however, the more detailed Fig. 6 shows an intrahepatic thrombus (level III) as requiring occlusion of the porta hepatis and division of the diaphragm for suprahepatic IVC control, thereby confirming the major hepatic veins as the intended landmark for level III. Figure 5 depicts an infrahepatic thrombus (level II) as requiring cranial retraction of the caudate lobe, which by definition requires division of the short hepatic 

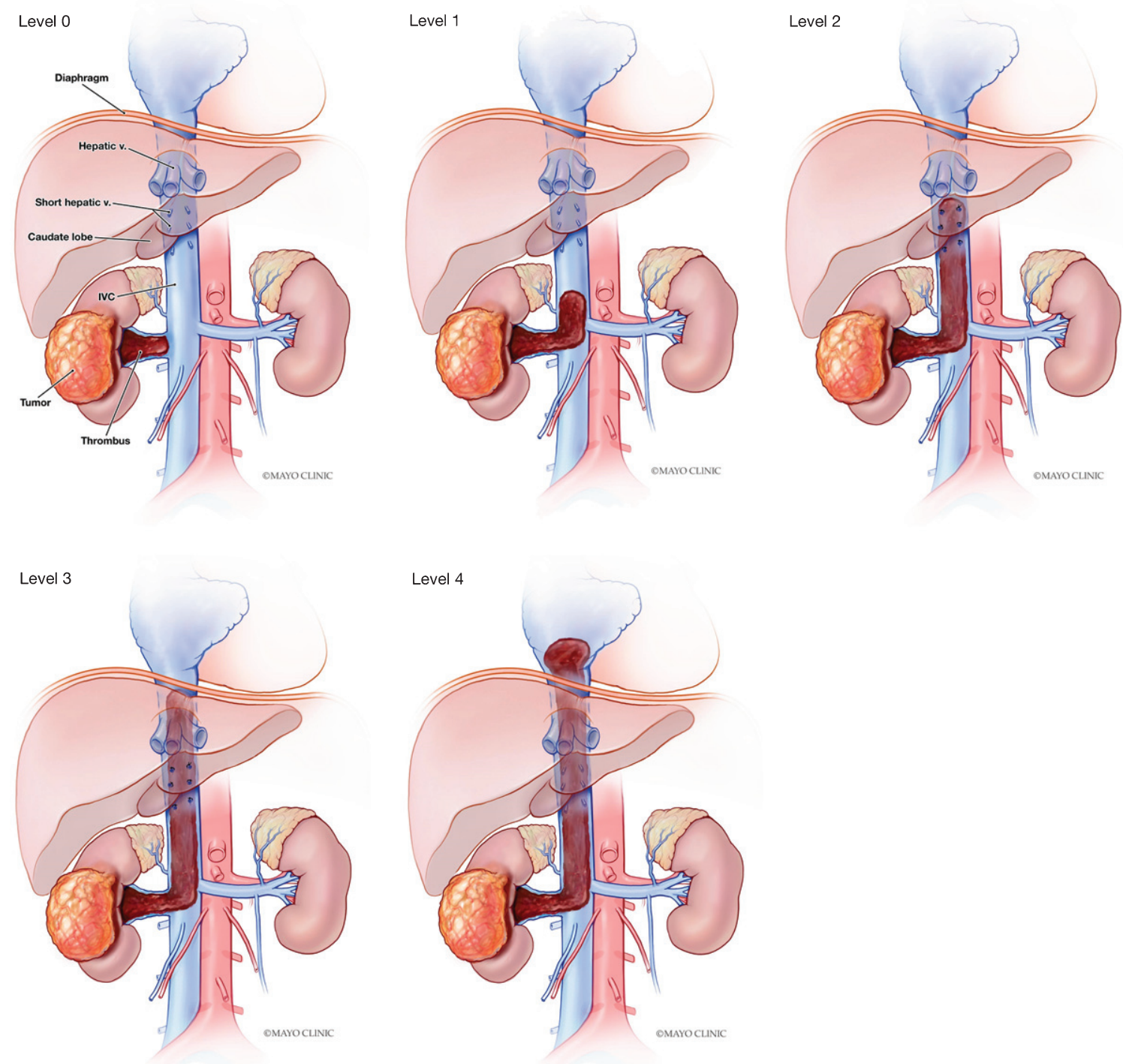

Fig. 1. - Proposed tumor thrombus levels. A) Level 0, thrombus within the renal vein; B) Level 1, thrombus into the IVC below the inferior margin of the caudate lobe; C) Level 2, thrombus above the inferior margin of the caudate lobe but below major hepatic veins; D) Level 3, thrombus at or above major hepatic veins but below diaphragm; E) Level 4, thrombus above diaphragm.

Table 2

Predictive ability of the current and modified thrombus classifications for perioperative outcomes

\begin{tabular}{lccc}
\hline & $\begin{array}{c}\text { Any 30-day } \\
\text { Complication } \\
A U C\end{array}$ & $\begin{array}{c}\text { Prolonged length } \\
\text { of Hospital stay } \\
A U C\end{array}$ & $\begin{array}{c}\text { High volume of } \\
\text { Blood transfused } \\
A U C\end{array}$ \\
\hline Training Cohort $(\mathrm{N}=166)$ & & & \\
$\quad$ Current Classification & 0.59 & 0.61 & 0.72 \\
$\quad$ Modified Classification & 0.58 & 0.61 & 0.72 \\
Validation Cohort $(\mathrm{N}=87)$ & & & 0.67 \\
$\quad$ Current Classification & 0.68 & 0.70 & 0.67 \\
Modified Classification & 0.67 & & \\
\hline
\end{tabular}

$A U C$ area under the curve. 
venous branches. Consistent with this interpretation, major hepatic veins have been used to delineate level III thrombi in subsequent publications from several high-volume centers [4]. This is reflected in the modified system proposed here (Fig. 1). In contrast, several recent robotic series have classified any thrombus above the inferior margin of the liver as a level III [2, 3]. A more granular classification system described by Ciancio, which assigns a subclassification of level III based on whether the thrombus is below, at the level of, or above the major hepatic veins may also be useful [5].

As minimally invasive surgical techniques are developed and refined for the management of RCC with IVC-TT, it is imperative that we critically evaluate both oncologic and quality of life outcomes of such novel techniques. This process is only possible via meticulous comparisons between surgical series. The modified classification developed herein did not improve predictive ability for perioperative outcomes and therefore doesn't warrant widespread use. Nevertheless, we advocate for standardization of the border of a level III thrombus as the major hepatic veins, consistent with the consensus from a multi-institutional open experience [4], in all future series of radical nephrectomy with IVC thrombectomy.

\section{ACKNOWLEDGMENTS}

The authors have no acknowledgements.

\section{FUNDING}

The authors report no funding.

\section{AUTHOR CONTRIBUTIONS}

BCL and TDL conceived of the presented idea. BCL, CML, JCC, PHS, SAB, RHT, and TDL refined the methodology planned the analyses. BCL, TDL, and TAP collected the primary data. CML performed statistical analysis. BCL, JCC, SAB, RHT supervised the analysis and data curation. CML, MT, PHS and TL drafted the manuscript. All authors critically revised the manuscript for important content and approved of the final manuscript.

\section{CONFLICT OF INTEREST}

All authors have nothing to disclose.

\section{REFERENCES}

[1] Neves RJ, Zincke H. Surgical treatment of renal cancer with vena cava extension. British journal of urology. 1987 May;59(5):390-5. PubMed PMID: 3594097.

[2] Abaza R, Shabsigh A, Castle E, Allaf M, Hu JC, Rogers C, et al. Multi-Institutional Experience with Robotic Nephrectomy with Inferior Vena Cava Tumor Thrombectomy. The Journal of urology. 2016 Apr;195(4 Pt 1):865-71. PubMed PMID: 26602891.

[3] Chopra S, Simone G, Metcalfe C, de Castro Abreu AL, Nabhani J, Ferriero M, et al. Robot-assisted Level II-III Inferior Vena Cava Tumor Thrombectomy: Step-by-Step Technique and 1-Year Outcomes. European urology. 2017 Aug;72(2):267-74. PubMed PMID: 27663048.

[4] Haddad AQ, Wood CG, Abel EJ, Krabbe LM, Darwish OM, Thompson RH, et al. Oncologic outcomes following surgical resection of renal cell carcinoma with inferior vena caval thrombus extending above the hepatic veins: a contemporary multicenter cohort. The Journal of urology. 2014 Oct;192(4):1050-6. PubMed PMID: 24704115.

[5] Ciancio G, Vaidya A, Savoie M, Soloway M. Management of renal cell carcinoma with level III thrombus in the inferior vena cava. The Journal of urology. 2002 Oct;168(4 Pt 1):1374-7. PubMed PMID: 12352396. 